\title{
CORIOCARCINOMA NO GESTACIONAL PRIMARIO DE OVARIO. A PROPÓSITO DE UN CASO Y REVISIÓN DE LA LITERATURA
}

\section{PRIMARY NONGESTATIONAL CHORIOCARCINOMA OF THE OVARY. ON THE SUBJECT OF A CASE AND REVIEW OF THE LITERATURE}



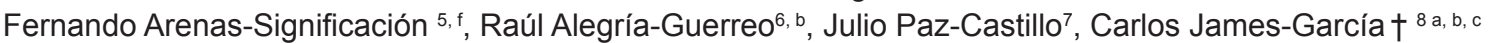

\begin{abstract}
RESUMEN
Se presenta el caso clínico de una paciente con diagnóstico de coriocarcinoma primario no gestacional de ovario que corresponde a una neoplasia maligna germinal. Asimismo, se revisa la literatura actualizada del tema haciendo énfasis en la biología molecular, el diagnóstico y el manejo oncológico inicial. Se desarrolló en el Hospital Nacional Dos de Mayo (HNDM) en el servicio de Ginecología Oncológica, Lima, Perú. Se registra el presente caso por su poca casuística en nuestro medio y por el importante valor del criterio oncológico en el manejo de masas anexiales ováricas.
\end{abstract}

Palabras clave: Coriocarcinoma primario no gestacional de ovario, neoplasia maligna germinal (Fuente: DeCS BIREME).

\begin{abstract}
We present the clinical case of a patient with a diagnosis of primary non-gestational ovarian choriocarcinoma corresponding to a germline malignant neoplasm. Likewise, the updated literature on the subject is reviewed, emphasizing molecular biology, diagnosis, and initial cancer management. It was developed at the Dos de Mayo National Hospital (HNDM) in the Oncology Gynecology Service, Lima, Peru. The present case is recorded due to its limited casuistry in our setting and due to the important value of oncological criteria in the management of adnexal ovarian masses.
\end{abstract}

Keywords: Primary non-gestational choriocarcinoma of ovarian, germline malignant neoplasm (Source: MeSH NLM).

\section{INTRODUCCIÓN}

El coriocarcinoma no gestacional de ovario, en sus siglas en inglés NGOC (Non-Gestacional Ovarian Choriocarcinoma), es una rara neoplasia maligna de las células germinales ${ }^{1}$. También, se le conoce como coriocarcinoma primario de ovario y aparece con mayor frecuencia en pre-púberes, según la mayoría de casos reportados en el mundo². Se calcula una incidencia alrededor de 0,6\% con una proporción del ovario izquierdo sobre el derecho con una razón de 13 en 7 demostrada en estudios observacionales ${ }^{3}$; asimismo, se pueden clasificar en puro y no puros ${ }^{2,4}$.

Los síntomas más comunes son: el sangrado transvaginal, la presencia de una masa anexial, el dolor abdominal, la pubertad precoz y las pruebas de embarazos positivas en la orina. Además, por esta última condición es fácil

1. Fellow(Subespecialidad) en Ginecología Oncológica. Universidad Peruana Cayetano Heredia, Hospital Nacional Edgardo Rebagliati Martins-EsSalud, Lima, Perú.

2. Jefe Servicio de Ginecología Oncológica del Hospital Nacional Dos de Mayo, Lima, Perú.

3. Subespecialista en Ginecología Oncológica, Hospital Nacional Edgardo Rebagliati Martins-EsSalud, Lima, Perú.

4. Médico Ginecóloga-Obstetra, Servicio de Reproducción Humana, Hospital Nacional Dos De Mayo, Lima, Perú.

5. Médico Radiólogo. Centro Nacional de Telemedicina. EsSalud, Lima, Perú

6. Jefe del Servicio de Obstetricia de Alto Riesgo, Hospital Nacional Guillermo Almenara Irigoyen (HNGAI) EsSalud, Lima, Perú.

7. Profesor Principal, Cátedra de Histología y Embriología Humana; y Genética Humana. Universidad Nacional Mayor de San Marcos (UNMSM), Facultad de Medicina Humana, San Fernando, Lima, Perú.

8. Doctor en Medicina Humana, Universidad Nacional Mayor de San Marcos, Facultad de Medicina Humana), Lima, Perú († 2021).

a. Miembro de la Sociedad Peruana de Ginecología Oncológica.

b. Miembro de la Sociedad Peruana de Ginecología y Obstetricia (SPOG).

c. Miembro Sociedad Peruana de Colposcopía y Patología del Tracto genital inferior.

d. Miembro de la Sociedad Española de Ginecología Oncológica del Grupo de investigación en Ovario.

e. Maestría en Investigación Clínica, Universidad San Martín de Porres.

f. Miembro de la Sociedad Peruana de Radiología.

g. Docente Universitario y actual Miembro del Comité de Ética en investigación de la Universidad Privada de Piura, Facultad de Medicina Humana. iD Código ORCID: https://orcid. org/0000-0002-9840-1886: Carlos Gonzales-Medina

Citar como: Gonzales-Medina C, Gálvez-Vizcarra S, Aguilar-Ramos P, Molina-Maldonado B, Arenas-Significación F, Alegría-Guerrero R, Paz-Castillo J, James-García C†. Coriocarcinoma no gestacional primario de ovario a propósito de un caso y revisión de la literatura. Rev Peru Investig Matern Perinat. 2021;10(1): 41-51

DOI https://doi.org/10.33421/inmp.2021228

Recibido: 15-02-2021 Aprobado: 31-03-2021 
confundir con un embarazo ectópico. Por ello, es difícil realizar el diagnóstico a partir de las características clínicas. Además, suele el tumor anexial suele manifestarse como una necrosis hemorrágica; y microscópicamente está constituido por sincitotrofoblasto y citotrofoblasto. En recientes investigaciones publicadas, se tienen datos sobre algunos de los factores pronósticos para orientar el tratamiento oncológico individualizado 5 .

El objetivo del presente artículo es mostrar a la comunidad médica peruana e internacional un caso clínico de coriocarcinoma primario de ovario (no gestacional) con su revisión teórica del tema. Además, el énfasis del presente estudio es desarrollar los datos actuales de la biología molecular de estos tumores y explicar el método de diagnóstico y el manejo oncológico inicial.

\section{CASO CLÍNICO}

Paciente mujer de 31 años, de nacionalidad peruana, natural de Junín, procedente de Huancayo, conviviente, con secundaria completa, con un nivel de instrucción secundaria completa y de ocupación ama de casa. No tenía antecedentes médicos de importancia reconocidos. Asimismo, no tenía antecedentes quirugicos, alergias o hábitos nocivos como el consumo de alcohol o drogas. Solo tenía el antecedente familiar de un contacto positivo por tuberculosis pulmonar activa hace veinte años. Dentro de sus antecedentes ginecobstétricos, tuvo una sola gestación a término, hace más de tres años, que culminó por vía vaginal sin complicaciones aparentes. Su menarquia se presentó a los catorce años, con un atraso menstrual de dos semanas antes del ingreso por emergencia. Del mismo modo, tenía como antecedente dos parejas sexuales a lo largo de su vida, con un inicio de su actividad sexual a los diecinueve años. En la historia clínica, no se halla el dato de algún estudio previo de citología cervical de rutina.

La paciente llegó a nuestra institución referida desde el Hospital Nacional II de Tarapoto del Ministerio de Salud, con un tiempo de evolución de ocho días caracterizado por cefalea holocraneana tipo opresiva de moderada intensidad, asociada a la disminución de la fuerza muscular en hemicuerpo derecho con predomino braquial, asociada a una parálisis de hemicara derecha. Dos días antes de su ingreso por emergencia, además cursó con melena (heces negras) con 2 a 3 deposiciones por día. Asimismo, existía el dato que los antecedentes de cefaleas eran de comportamiento ocasional incluso desde los 15 años de edad, sin auras o gatillos desencadenantes. Al examen físico de su ingreso por emergencia, se describe una presión arterial en 100/64 mmHg, su frecuencia cardiaca en 80 latidos por minutos, su frecuencia respiratoria en 22 respiraciones por minuto, su temperatura en $36,7^{\circ} \mathrm{C}$, con una saturación de $\mathrm{O}_{2}$ del $98 \%$ y un peso de $62 \mathrm{~kg}$. La paciente se hallaba, despierta, ventilaba espontáneamente, tenía palidez marcada, parálisis facial derecha, leve dolor en hipogastrio, una evaluación de Glasgow en 15, se hallaba lúcida, orientada aun en tiempo, espacio y persona. Además, presentaba hemiparesia del cuerpo derecho con predomino en miembros superiores; y llegó a la emergencia recibiendo dos transfusiones sanguíneas previas. En la emergencia de medicina ingresó como probable gestación, dado que no quedaba claro el tiempo del atraso menstrual; $y$, tenía un examen en orina cualitativo positivo para la subunidad B hCG. Llegó con Hb: 5 g/dl, leucocitos: 4600 $\mathrm{mil} / \mathrm{mL}$, plaquetas: $330000 \mathrm{mil} / \mathrm{mL}$, con una tomografía axial computarizada de su centro de origen, en la que se describía una imagen redondeada mixta, a nivel de región frontoparietal izquierda de $40 \mathrm{~mm} \times 50 \mathrm{~mm}$ y otra imagen isodensa redondeada de $10 \mathrm{~mm} \times 10 \mathrm{~mm}$, a nivel occipital derecha. Por ello, en la referencia del Hospital de Tarapoto II se consideró los siguientes diagnósticos: 1) Gestante de aproximadamente \pm 3 semanas por última regla con aparente prueba positiva cualitativa, 2) Metástasis cerebral por probable cáncer de pulmón izquierdo solitario versus segundo primario, 3) Hemorragia digestiva alta, 4) Anemia severa y 5) Nódulo en pulmón izquierdo solitario a descartar secundarismo versus neoplasia primaria o metastásica de segundo primario no identificado. En la anamnesis indirecta (del ingreso por emergencia HNDM) se registra que la paciente tiene un tiempo de enfermedad de 2 semanas, de forma de inicio brusco, con curso progresivo, caracterizado principalmente por cefalea holocraneana de predomino occipital de moderada intensidad, asociada a hemiparesia derecha y parálisis facial. No se precisa el tiempo de retraso menstrual, pero se consideró como gestante al inicio dado que tenía una prueba positiva por ser una mujer en edad fértil. Asimismo, se documentó en la historia clínica melena en 2 a 3 oportunidades. Al examen físico de emergencia, la paciente se hallaba confusa, con hemiparesia derecha, parálisis facial y hemiparesia derecha a predominio braquial. Se consideró como diagnósticos de ingreso: 1) Proceso expansivo frontoparietal a descartar neoplasia maligna cerebral versus de origen metastasico, 2) Hemorragia digestiva alta no activa, 3) Anemia severa, 4) Trastorno hidroelectrolítico: Hiponatremia leve, 4) Descartar gestación inicial y 5) Nódulo pulmonar por historia clínica. Por lo tanto, se planteó la evaluación por neurocirugía de emergencia y preparar pre quirúrgicos. La radiografía de tórax antero-posterior mostró nódulos con densidad de partes blandas de márgenes definidos lobulados, proyectados a nivel de la base de campo pulmonar derecho y a nivel parahiliar en el campo pulmonar izquierdo. Estos hallazgos están relacionados a secundarismo pulmonar (Figura $1 \mathrm{~A}$ ). En una primera evaluación por neurocirugía de emergencia, se consideró el manejo médico antiedema con dexametasona 8mg endovenoso cada 8 horas. Y en una segunda evaluación, a la hora de la primera, la paciente cursó sin mejora médica y se evidenció en las cisternas cerebrales obturación anatómica, con signos de hipertensión endocraneana. Por lo que se decidió intervenir quirúrgicamente por sala de operaciones de emergencia. 
La cirugía fue una craneotomía frontoparietal izquierda con resección total del tumor frontal, en la que se describió como hallazgos principales macroscópicos a nivel cerebral un tejido de color negruzco de forma irregular en el que a nivel microscópico se consideró como primera posibilidad un coriocarcinoma metastasico (Figura 2 A y 2a). Posteriormente, ingresó a la unidad cuidados críticos y cursó con una buena evolución postoperatoria. Ingresó a hospitalización del servicio de Neurocirugía con evolución favorable postoperatoria. Sin embargo, presentó un cuadro de hemorragia digestiva baja activa. Por otro lado, en la tomografía computarizada (TC) de encéfalo postoperatoria y sin contraste, se halló múltiples imágenes nodulares subcorticales a nivel supratentorial en ambos hemisferios cerebrales, las cuales mostraban hiperdensidad espontánea que sugirió una naturaleza hemorrágica, por lo que los hallazgos se catalogaron en relación a las metástasis hemorrágicas. Asimismo, se evidenció hipodensidad difusa de la sustancia blanca de la corona radiada y de los centros semiovales que acentuaba la diferenciación de la sustancia gris y la sustancia blanca, en relación con un edema vasogénico intralesional (Figura $1 \mathrm{~B}$ ).

El cuadro de la hemorragia digestiva fue monitorizado por el servicio de gastroenterología con un estudio endoscópico diferido al parecer por limitaciones de la paciente por su estado neurocritico. Sin embargo, se alcanzó a determinar que no había evidencia de sangrado activo en las zonas de evaluación endoscópica, dado que existían zonas de lesiones rojo vinosas en el cuerpo gástrico de $4 \mathrm{~mm}$ y $10 \mathrm{~mm}$, sin sangrado activo de probable origen metastásico, así como lesiones de similares características en la segunda porción del duodeno y el motivo de la no realización de las biopsias de las lesiones fue una plaquetopenia moderada previa. Frente a la persistencia del cuadro hemorrágico, su evolución en los siguientes días fue estacionaria. De tal modo, que en los días sucesivos presentó una palidez marcada y se agregó súbitamente un dolor abdominal moderado a nivel de hipogastro no irradiado. Además, se evidenció una caída de la hemoglobina, incluso post trasfusión sanguínea. En esta circunstancia fue interconsultada por el servicio de Oncología Ginecológica con los estudios de ecografía transvaginal actualizado, sin evidencia de embarazo intrauterino, con anexos aparentemente sin alteraciones. $Y$ tuvo una subunidad B hCG cuantitativa en $117279 \mathrm{UI} / \mathrm{ml}$. Asimismo, durante la evaluación llamó la atención el dolor abdominal bajo intenso. Y al examen clínico, se describió un marcado dolor a la movilización cervical, no sangrado activo transvaginal, y el fondo de Saco de Douglas ocupado en cavidad pélvica.

Por ello, por la caída brusca del hematocrito, la subunidad beta positiva, la clínica de dolor abdominal bajo de moderada intensidad, y que la paciente se halló hemodinamicamente inestable, sin evidencia aparente de embarazo intrauterino se decide intervenir quirúrgicamente de emergencia con una laparotomía exploratoria por probable embarazo ectópico complicado. Se realizó solo una biopsia en cuña de del ovario afectado. Dentro de los hallazgos intraoperatorios se describió un útero en anteversoflexión de $8 \times 5 \times 3 \mathrm{~cm}$ de superficie regular sin lesiones en su superficie. La trompa derecha y el ovario derecho se documentaron con características aparentemente normales. Asimismo, en la trompa izquierda se describe una tumoración de $1 \mathrm{~cm}$ sobreelevada de aspecto violáceo, se tomó una muestra por resección en cuña no sangrante. $Y$ a nivel de asas intestinales colónicas se evidenció un aparente contenido hemático. También se describió el apéndice cecal sin alteraciones, con líquido seroso escaso en cavidad peritoneal y ausencia de sangrado intraoperatorio. El estudio anatomopatologico de la resección en cuña evidenció la presencia de una neoplasia maligna dispuesta en un patrón plexiforme bifásico compuesta por células de sincitiotrofoblasto multinucleadas con citoplasma eosinofilo denso y núcleos picnoticos. Así como, un trofoblasto intermedio y citotrofoblasto con cromatina granular irregular y nucléolos prominentes. Por lo que existió extensas áreas de necrosis y hemorragias (Figura 2 A-a).
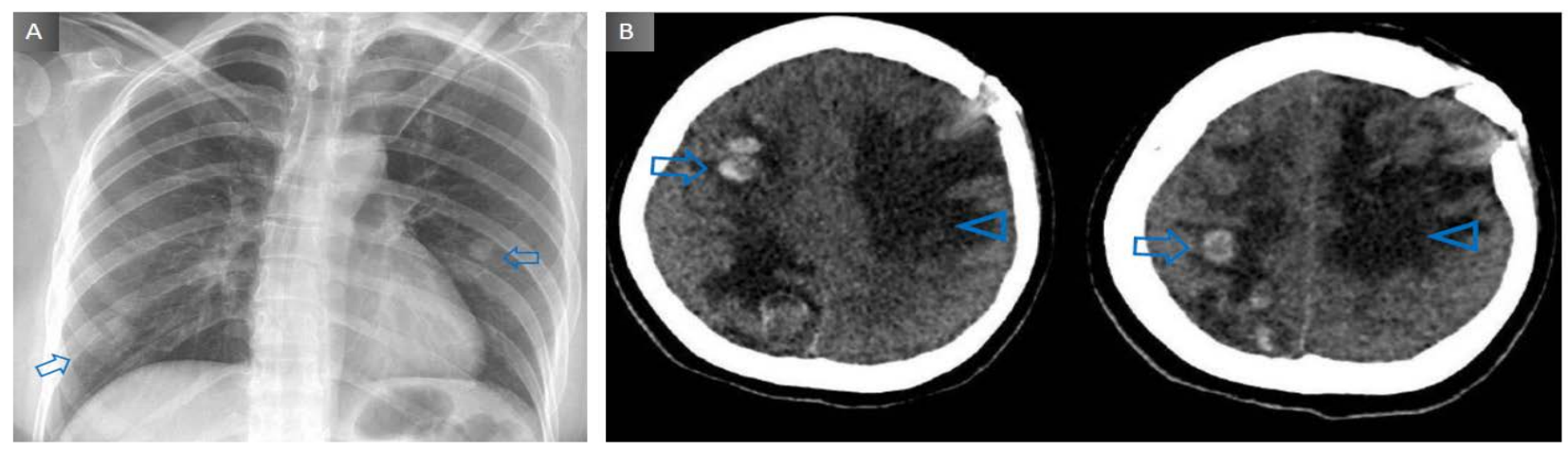

Figura 1. Estudio imagenológico: (A) Radiografía de tórax antero-posterior: muestra nódulos proyectados en campos pulmonares en relación con secundarismo pulmonar (flechas azules). (B) TC de encéfalo sin contraste: muestra nódulos hiperdensos subcorticales bilaterales en relación con metástasis hemorrágicas (flechas), asociado a edema vasogénico difuso (cabezas de flechas azules). 



Figura 2. Estudio anatomopatológico microscópico en coloración Hematoxilina-Eosina: (A-20X) y (a- 40X) Tejido cerebral con presencia de hallazgos compatibles con un coriocarcinoma metastásico como primera posibilidad. (B-20X) y (b-40X) corresponde a biopsia en cuña de ovario en la que se describe una neoplasia maligna con un patrón plexiforme bifásico compuesta por células de sincitiotrofoblasto multinucleadas con citoplasma eosinofilo denso y núcleos picnoticos. Así como, un trofoblasto intermedio y citotrofoblasto con cromatina granular irregular y nucléolos prominentes. Se concluye como tumor de células germinales: Coriocarcinoma puro primario de ovario.

Posterior a la laparotomía ingresó a una unidad de hospitalización en medicina interna y oncológica de nuestra institución. Fue evaluada por distintas especialidades quienes brindaron sus puntos de vista en las interconsultas respectivas: Medicina intensiva sugirió el manejo en relación al medio interno y hemoderivados. Neurocirugía opinó el alta por su especialidad y manejo por oncología médica, Hematología: sugirió manejo en relación al uso de hemoderivados. Gastroenterología realizó una endoscopía y recomendó una reevaluación con mejoría hematológica. Medicina de Rehabilitación sugirió terapia física y terapia de lenguaje en 3 sesiones. Oncología médica recién evaluó a la paciente a los ocho días post laparotomía sugiriendo el diagnóstico de Tumor Germinal de Alto Grado Primario de Línea Media solicitando una TC Cebrero-Tóraco-Abdómino-Pélvica con contraste, con dosaje de $\beta$-HCG, perfil hepático y perfil de coagulación como parte del seguimiento. Y recomendó que la paciente necesitaba un manejo sistémico urgente. Sin embargo, la paciente en la unidad de medicina interna cursó con hemorragias digestivas y solo se dio manejo de soporte, y no se inició otra terapéutica adicional. Los valores de $\beta$-HCG cuantitativo durante toda su estancia hospitalaria se duplicaba rápidamente en función de los días (Figura 3). Su última analítica, presentó un perfil hepático con TGO, TGP Normal y una albumina sérica en $1,92 \mathrm{mg} / \mathrm{dl}$. Lamentablemente, por el estado neurocrítico de la paciente y los cuadros hemodinámicos alterados, falleció de un paro cardiaco. No se logró realizar autopsia



Evaluación en el tiempo de los niveles de BHCG

Figura 3. Evolución de los niveles de $\beta$-HCG: Corresponde al seguimiento de los niveles de $\beta$-HCG medido en $U I / m L$, según el momento de las intervenciones quirúrgicas. Se evidencia claramente que durante el monitoreo de los valores existía una tendencia marcadamente elevada. 
clínica por falta de autorización familiar. Sin embargo, el caso clínico se manejó como un tumor germinal de alto grado primario de ovario.

\section{DISCUSIÓN}

Los tumores ováricos malignos se clasifican clásicamente en tres categorías principales según las estructuras anatómicas de las cuales se originan ${ }^{6}$. Estas incluyen las neoplasias de origen epitelial, estromal y germinal. Las neoplasias epiteliales constituyen cerca del 90 al 95\% de los tumores malignos de las gónadas femeninas ${ }^{7}$. Y alrededor del 5 al $10 \%$ restante constituyen los tumores ováricos del estroma de los cordones sexuales y de las células germinales ${ }^{8}$. Éstos últimos tienen cualidades únicas que requieren una estrategia diagnóstica y terapéutica individualizada. Y, por lo tanto, constituyen en la práctica clínica un verdadero reto diagnóstico ${ }^{2,8}$.

Los orígenes de los tumores ováricos germinales constituyen los elementos primordiales de las gónadas femeninas. De hecho, el teratoma quístico maduro, denominado quiste dermoide constituye el subtipo histológico más frecuente de todas las neoplasias germinales alcanzando alrededor del 95\% de los casos. Además, el curso de su comportamiento clínico es principalmente benigno ${ }^{9}$. En ese sentido, la National Comprehensive Cancer Network (NCCN) de acuerdo con la clasificación actual de la Organización Mundial de la Salud (OMS) considera en la actualidad a estos tumores como parte del grupo de subtipos histológicos por neoplasias malignas ováricas ${ }^{10}$. Además del teratoma maduro, los otros tumores germinales incluyen: el tumor del saco vitelino, el disgerminoma, el carcinoma embrionario, el teratoma inmaduro, el tumor mixto de células germinales y el coriocarcinoma no gestacional ${ }^{11}$. Éste último, también denominado coriocarcinoma primario de ovario, para diferenciarlo correctamente del grupo de las neoplasias trofoblasticas gestacionales. Una característica propia de esta neoplasia maligna de alto grado es su carácter mortal y agresivo. Por lo tanto, es importante señalar que todos los cánceres mencionados anteriormente tienen un comportamiento maligno con excepción del teratoma maduro ${ }^{12}$.

En vista de su aparición poco frecuente, es interesante entender su biología molecular y fisiopatología para reconocer las manifestaciones clínicas de esta neoplasia en las pacientes ${ }^{12}$. Por ello, resulta muy importante reconocer que desde el conocimiento de la citodiferenciacion inicial de la gónada indiferenciada es posible entender estos mecanismos subyacentes a su biología molecular ${ }^{5,13}$.

La gónada primitiva (indiferenciada) aparece alrededor de la sexta semana de desarrollo embrionario como parte del proceso embriológico del desarrollo gonadal femenino y masculino. De acuerdo a la ausencia de la expresión de la proteína SRY, se establece el desarrollo femenino.
Esta expresión es garantizada por la inactivación del gen SRY que se halla en la región codificante determinante del sexo en el brazo corto del cromosoma Yp11, dado que el producto proteico de este gen es un factor de transcripción que inicia la cascada de activación corriente abajo para el desarrollo gonadal masculino ${ }^{14}$.

Debemos comprender todos los mecanismos moleculares subyacentes implicados en la fisiopatología de este cáncer. Si bien el origen de las gónadas femeninas se remonta a las estructuras embrionarias derivadas de la gónada indiferente, existe un momento previo antes de este fenómeno trascendental ${ }^{15}$. $\mathrm{Y}$ es que el origen primario de los gametos deriva de las células germinales primordiales (gonocitos) que se forman en el epiblasto durante la segunda semana del desarrollo embrionario y estas células se desplazan hacia la pared posterior del saco vitelino muy cercano al alantoides. Luego de allí recién se desplazan hacia las gónadas indiferentes, en las cuales llegan hacia el final de la quinta semana en su proceso migratorio.

Pasando desde intestino primitivo posterior y pasando por el mesenterio a la región dorsal para albergarse en la sexta semana en los cordones sexuales desarrollados en las gónadas a partir de la cresta urogenital de los mesonefros. El número de estas células se incrementa por divisiones mitóticas durante la migración celular, y también cuando llegan a la gónada indiferente para desarrollarse ${ }^{16}$. Cuando falla este mecanismo de migración celular, por una orden de los genes del desarrollo, se pueden producir alteraciones muy importantes anatómicas y mecanismos de formación o inactivación de genes supresores o alteraciones de la reparación del ADN.

La metilación del ADN es un proceso que ocurre durante la gametogénesis y el desarrollo previo a la implantación. En la gametogénesis, las células germinales primordiales pasan por un proceso conocido como la desmetilación del ADN, incluidos los genes improntados en los que se generan los mecanismos de metilación de novo y ganancia de improntas ${ }^{12}$. En embriones preimplantacionales, la desmetilación del ADN ocurre por segunda vez, aunque los genes improntados y algunas secuencias repetidas carecen de desmetilación. Después de la implantación, se produce la remetilación en mayor medida en el linaje de células embrionarias en comparación con los linajes extraembrionarios. Se cree que los tumores de las células germinales testiculares derivan de una célula germinal primordial en una etapa del desarrollo en la que está hipometilada y muy lejos del mecanismo de impronta genómica. La metilación del ADN aumenta con la diferenciación del tumor de células germinales testiculares. Por ello, el teratoma de tipo embrionario y el tumor del saco vitelino de tipo extraembrionario (YST) y coriocarcinoma muestran más procesos de metilación que el carcinoma embrionario indiferenciado ${ }^{12,17}$ (Figura 4). 




Figura 4. Estado de metilación del ADN. Los niveles de metilación del ADN en las diferentes etapas del desarrollo embriológico y en diferentes tipos de tumores celulares. Traducido y modificado por el autor de la Figura 6 del artículo original con permiso de Blackwell Publishing Ltd (18).

Los estudios citogenéticos y moleculares, predominantemente sobre el coriocarcinoma gestacional, revelan el gran impacto de los oncogenes, los genes supresores de tumores y los genes de impronta en su patogénesis. De tal modo que se cree que el tumor de células germinales gonadales surge de una célula germinal primordial, que está hipometilada y carece de impronta. La metilación del ADN aumenta con la diferenciación normal de cualquier línea celular ${ }^{18}$.

Entre los tumores de células germinales, el coriocarcinoma extraembrionario similar a la placenta presenta un nivel más alto de metilación que el seminoma o el carcinoma embrionario indiferenciado, pero se expresa con un nivel más bajo en comparación con los teratomas bien diferenciados ${ }^{18}$. En el coriocarcinoma gonadal se ha informado una reducción de la expresión e hipermetilación de RASSF1A y PRSS21, aunque el tamaño de la muestra fue pequeño, debido a la rareza de la lesión constituye una evidencia científica importante para conocer los mecanismos moleculares propios de esta neoplasia a nivel ovárico ${ }^{17}$. La hibridación genómica comparativa de matriz integrada $(\mathrm{CGH})$ de todo el genoma y el perfil de expresión génica de tumores de células germinales testiculares, incluidos los coriocarcinomas, se encontraron, como se esperaba, que la ganancia del brazo cromosómico 12p era la aberración más común ${ }^{19,20}$. Se detectó sobreexpresión de genes relacionados con células madre, algunos de ellos localizados en el cromosoma 12 $p^{19,21}$. Además, se demostró una diferencia en el perfil de expresión, incluidos los genes relacionados con las células madre NANOG, STELLA y GDF3, entre seminomas y no seminomas de diversos tipos histológicos ${ }^{19}$. La ganancia de $12 p$ se considera funcionalmente relevante en la activación de genes relacionados con las células madre y en el estado alterado de las funciones de las células madre ${ }^{19}$.

Los estudios de perfil de expresión de todo el genoma también demostraron que las transcripciones de genes asociados con el desarrollo placentario y los trofoblastos se regulaban positivamente de manera diferencial en el coriocarcinoma entre los diversos tipos de tumores de células germinales gonadales ${ }^{19,22}$. En particular, se encontró que las subunidades de la gonadotropina coriónica (CGB5 y CGA) estaban sobreexpresadas de manera constante ${ }^{21,22}$. De hecho, entre las transcripciones predictivas identificadas a partir de los tumores mixtos de células germinales, se encontró que la beta hCG se asignaba con precisión a los tumores en los que el coriocarcinoma es el tumor predominante ${ }^{22}$. Asimismo, la determinación del origen gestacional o no gestacional de un coriocarcinoma tiene un impacto relevante en el curso clínico y el manejo de las pacientes.

El coriocarcinoma no gestacional parece tener un pronóstico más precario que el coriocarcinoma gestacional ${ }^{47}$. La tasa de supervivencia acumulada para el coriocarcinoma gestacional ampliamente diseminado sigue siendo aproximadamente del $80 \%$, mientras que las pacientes con tumores extragonadales de células germinales tienen una tasa de supervivencia menor del $40 \%$. Para las pacientes con coriocarcinoma gestacional, la quimioterapia se diseña de acuerdo con la puntuación pronóstica de cada paciente y generalmente es curativa ${ }^{12,47}$. 
Tabla 1. Marcadores tumorales en lesiones trofoblasticas según los oncogenes, genes supresores de tumores y reguladores del ciclo celular, muerte celular y senescencia, adhesión, motilidad e invasión celular, genes improntados y células madres con factores de transcripción

\begin{tabular}{|c|c|c|}
\hline $\begin{array}{c}\text { Eventos genéticos y moleculares } \\
\text { involucrados }\end{array}$ & $\begin{array}{c}\text { Nivel relativo en el Coriocarcinoma comparado } \\
\text { con el tejido placentario }\end{array}$ & Referencias \\
\hline \multicolumn{3}{|c|}{ Oncogenes, genes supresores de tumores y reguladores del ciclo celular } \\
\hline p53 & Incrementada expresión & (23) \\
\hline p21 & Incrementada expresion & (24) \\
\hline Mdm-2 & Incrementada expresion & $(24,25)$ \\
\hline $\mathrm{Rb}$ & Incrementada expresión & $(25,26)$ \\
\hline DOC-2/hDab2 & Disminuida expresión & (26) \\
\hline c-erb-B-2 & Incrementada expresión & $(27)$ \\
\hline Cyclin E & Incrementada expresión & (28) \\
\hline Maspin & Disminuida expresión & (29) \\
\hline NECC1 & Disminuida expresión & (30) \\
\hline RASSF1A & Disminuida expresión & (31) \\
\hline Promotor de la hipermetilación de p16. & Incrementada expresión & \\
\hline \multicolumn{3}{|l|}{ Muerte celular y senescencia } \\
\hline Telomerasa & Incrementada expresión & $(32)$ \\
\hline Apoptosis & Incrementada expresión & (33) \\
\hline $\mathrm{Bcl}-2$ & Incrementada expresión & (34) \\
\hline Ki67 & Incrementada expresión & (23) \\
\hline Mcm7 & Incrementada expresión & (35) \\
\hline Id1 & Incrementada expresión & (35) \\
\hline Caspasa & Disminuida expresión & $(36)$ \\
\hline \multicolumn{3}{|l|}{ Adhesión, motilidad e invasión celular } \\
\hline E-caderina/ $\beta$-catenina & Disminuida expresión & (37) \\
\hline MMPs* & Incrementada expresión & (38) \\
\hline TIMPs* & Disminuida expresión & (39) \\
\hline Promotor de la metilacion de TIMP* & Incrementada expresión & (39) \\
\hline \multicolumn{3}{|l|}{ Promotor de la hipermitalacion } \\
\hline de E-caderina & Incrementada expresión & (39) \\
\hline Mel-CAM* & Incrementada expresión & $(40)$ \\
\hline \multicolumn{3}{|l|}{ Genes improntados } \\
\hline $\mathrm{H} 19$ & Incrementada expresión & $(41)$ \\
\hline IGF2 & Disminuida expresión & $(41)$ \\
\hline P57/ KIP2 & Disminuida expresión & $(42)$ \\
\hline \multicolumn{3}{|l|}{ Células madres y factores de transcripción } \\
\hline NANOG & Incrementada expresión & $(43)$ \\
\hline Oct-04 & Disminuida expresión & $(44)$ \\
\hline SOX2 & Disminuida expresión & (45) \\
\hline STAT3 activado & Incrementada expresión & $(46)$ \\
\hline Hipermetilación de OCT4 & Incrementada expresión & (44) \\
\hline Promotor de la metilacion de SOX2 & Incrementada expresión & $(45)$ \\
\hline
\end{tabular}

* CAM: Cell adhesion molecule( Moleculas de adhesion celular); MMP: Matrix metalloproteinase(Metaloproteinasas de la matrix); TIMP: Tissue inhibitor of metalloproteinase (Inhibitor tisular de las proteinasa)

Los tumores de células germinales de ovario que producen hCG también se pueden curar con quimioterapia, pero se requieren diferentes regímenes farmacológicos $\mathrm{y}$, por lo general, está indicada la cirugía. Además, para diseñar la quimioterapia se utiliza el estadio de la enfermedad en lugar del sistema de evaluación pronóstica. Las pacientes con carcinoma somático con diferenciación trofoblástica también pueden responder a la quimioterapia, aunque la posibilidad de curación es mucho menor ${ }^{11,12,47}$.

No hay diferencia morfológica entre el coriocarcinoma gestacional y el coriocarcinoma de origen de células germinales. La historia clínica puede ayudar en el diagnóstico diferencial. Si la paciente es premenárquica o si están presentes otros elementos tumorales de células germinales, se puede determinar que el coriocarcinoma es del tipo de células germinales ${ }^{47,48}$. El coriocarcinoma es de origen gestacional si un componente paterno puede identificarse mediante análisis de ADN o tipificación de $\mathrm{HLA}^{49}$. Por lo tanto, el diagnóstico genético molecular, por ejemplo, el genotipado de microsatélites fluorescentes, puede usarse para evaluar el origen genético de tumores productores de beta hCG con diferenciación trofoblástica ${ }^{12,49}$. 
Debido a su coexistencia con otros tumores de células germinales o el carcinoma somático asociado, la aparición del coriocarcinoma no gestacional puede ser más variable, pero suele encontrarse un nódulo hemorrágico. Histológicamente, el patrón bifásico es idéntico al de los casos gestacionales. El coriocarcinoma que acompaña a un carcinoma somático puede mostrar una transición entre el coriocarcinoma y el adenocarcinoma escamoso, transicional o el adenocarcinoma ${ }^{50}$.

Los tumores germinales constituyen el segundo grupo más grande de los tumores de ovario alrededor del 20\% y pueden presentarse a cualquier edad. La mayor incidencia se observa en la segunda década de la vida y en las mujeres adultas la gran mayoría son benignos (teratomas quísticos maduros). En el grupo de los tumores malignos el disgerminoma ocupa el primer lugar representando el $50 \%$ de los casos. Además, se sabe que el $20 \%$ se presenta durante el embarazo y el $80 \%$ de los casos en mujeres menores de 30 años ${ }^{51}$.

De todos modos, para conocimiento del clínico es menester reconocer que la forma de presentación habitual en la práctica diaria de los tumores de la línea germinal contrasta de los tumores de origen epitelial. Por lo tanto, es muy importante realizar una adecuada anamnesis y una prolija evaluación clinica. Los exámenes auxiliares permitirán añadir mayor información a la sospecha clinica, pero es necesario ejercitar una buena correlación clínicopatológica en el método nosológico de la enfermedad.

Frente a una masa anexial en el contexto de una valoración bioquímica con el marcador CA125 muy elevado, la sospecha clinica debe ser la pieza angular para el manejo correcto del caso clínico. Desde el punto de vista del manejo oncológico resulta muy interesante su identificación precoz y el tratamiento oportuno individualizado. Porque, del reconocimiento de los factores pronósticos se determinará una conducta tanto el manejo medico oncológico como el manejo con criterio oncológico-quirúrgico ${ }^{48,51}$.

En un estudio publicado en abril del 2020 realizado en el Hospital Qilu de la Universidad de Shandong de la República Popular de China ${ }^{5}$ se identificó a 39 pacientes de un total de 36 estudios publicados, mediante una metodología de revisión bibliométrica, usando una serie de términos de búsqueda en PubMed, en la biblioteca Cochrane y en Google Scholar con el objetivo de determinar los casos publicados entre el 1 de enero de 1967 y el 31 de julio de 2018. Los términos de búsqueda en mención (con operadores booleanos) fueron los siguientes: (Ovary [Mesh]) OR Ovarian OR oophoron AND Choriocarcinoma, Non-gestational [Mesh] OR Choriocarcinoma, Non gestational OR Choriocarcinomas, Non-gestational OR Non-gestational Choriocarcinomas OR Non-gestational Choriocarcinoma OR Non gestational Choriocarcinoma. Con estos términos de búsqueda, dicha investigación tuvo como objetivo principal analizar los factores pronósticos del NGOC y resumir sus características clínico-patológicas para orientar el tratamiento individualizado.

Como hallazgos principales destacan los siguientes: la mediana de edad fue de 30 años con un rango de 12 a 65 años; la incidencia máxima fue en la edad comprendida entre los 12 a 25 años; la mediana de seguimiento de los casos fue de 20,3 meses (rango 1-84 meses). Además, se documentó por las características agresivas de esta neoplasia que 9 (23\%) pacientes fallecieron, 24 (62\%) pacientes vivieron y solo $6(15 \%)$ se perdieron durante el seguimiento. Tras el análisis univariado, encontraron los autores que la edad tuvo un impacto pobre en la supervivencia general (SG) en NGOC, HR - 0.05, IC del $95 \%[0.11-<0.00]^{5}$. El coriocarcinoma no gestacional de ovario tipo puro tiene una supervivencia general (SG) superior que el de tipo mixto (HR - 2,62, IC del 95\% - [4,57 - 0,66]. Desde el punto de vista quirúrgico la resección $\mathrm{R} 0$ es un factor de buen pronóstico para la supervivencia general, con HR 2,96, IC 95\% [0,71-5,21]. Los médicos deben intentar lograr la resección R0 para mejorar el pronóstico de los pacientes con coriocarcinoma no gestacional de ovario tipo puro, incluso entre los pacientes avanzados $^{52}$.

Se deben realizar más estudios para identificar genes supresores de tumores y oncogenes en tales loci para mejorar nuestra comprensión de los mecanismos genéticos que gobiernan el coriocarcinoma. Se puede considerar que el desarrollo de coriocarcinomas gestacionales y gonadales recapitula la diferenciación embriológica con un estado similar de impronta y metilación. El análisis de los efectos funcionales de genes expresados diferencialmente y factores de transcripción de células madre centrales en estos tumores pueden arrojar indirectamente más horizontes sobre el desarrollo embriológico y placentario.

Con la identificación de oncogenes y genes supresores de tumores en el coriocarcinoma, se puede desarrollar una nueva terapia molecular dirigida. Por ejemplo, se han demostrado la expresión aberrante y los efectos celulares de EGF-R y factores de transcripción de células madre como NANOG y STAT3. Debe aclararse el mecanismo molecular que subyace a tales efectos celulares. La terapia dirigida anti-EGF-R o anti-NANOG también puede explorarse para el tratamiento del coriocarcinoma refractario a la terapia convencional, independientemente de su origen ${ }^{43}$. Los estudios genéticos apoyan la posibilidad que los coriocarcinomas surgen en asociación con el carcinoma somático y comparten un origen común de células madre como el carcinoma somático ${ }^{43,52}$. Se necesitan más estudios para investigar el mecanismo que provoca la desdiferenciación dado que este fenómeno ocurre raramente $\mathrm{e}^{12,52}$.

El diagnóstico de coriocarcinoma extrauterino primario es un desafío. No existe una manifestación clínica específica 
atribuible al coriocarcinoma de ovario. Sin embargo, su presentación puede simular otras patologías como la torsión ovárica y el embarazo ectópico ${ }^{53}$. El coriocarcinoma produce una enorme cantidad de gonadotropina coriónica humana beta $(\beta-H C G)$. El valor varía de tres a 100 veces más que un embarazo normal. Las mediciones en serie del nivel de $\beta$-HCG son de gran valor diagnóstico. También mide la respuesta al tratamiento ${ }^{54}$. Además, los sitios potenciales donde el coriocarcinoma puede hacer metástasis incluyen los anexos uterinos, la cavidad pélvica, los pulmones, el cerebro y el hígado. En raras ocasiones, también puede hacer metástasis en el feto a través del tejido placentario ${ }^{55}$. La metástasis suele ser hemorrágica debido a la capacidad innata de las células trofoblásticas para invadir y erosionar las paredes de los vasos $^{55}$.

La quimioterapia es el tratamiento de elección para el coriocarcinoma, incluso en la enfermedad metastásica. Tiene una tasa de curación de aproximadamente el $95 \%{ }^{51,55}$.

Actualmente, cuatro a seis ciclos de cisplatino, etopósido y bleomicina (PEB) como terapia convencional son el estándar terapéutico para el tumor de células germinales de ovario diseminado (TCG) con excelente actividad y toxicidad aceptable. Asimismo, se conoce que la supresión ovárica temporal con agonistas $\mathrm{GnRH}$ durante la quimioterapia no aumenta significativamente las posibilidades de reanudación de la menstruación en pacientes jóvenes con neoplasias germinales malignas de ovario ${ }^{56}$. Recomendamos la evaluación temprana de todas las pacientes por carcinoma de ovario que presente hemorragia per vaginum. El diagnóstico temprano puede ayudar a un mejor manejo de los pacientes, así como a la prevención de la enfermedad metastásica.

Esta publicación corresponde a un caso originario de nuestro país y constituye el primer caso publicado en español en nuestro medio sobre un coriocarcinoma primario puro de ovario en una mujer en edad fértil.

Como conclusión es importante señalar el valor diagnóstico y las estrategias actuales de la biología molecular, para perfeccionar el manejo de estos cuadros clínicos de comportamiento agresivo, considerando que la cirugía oncológica tiene un rol importante en el pronóstico y manejo de esta enfermedad.

Financiamiento. Autofinanciado.

Declaración de conflictos de intereses: Los autores declaran no tener conflicto de intereses.

Agradecimientos. Queremos agradecer a la familia del Dr Carlos James-García $(\dagger)$ quien fue un descatado profesional médico, Doctor en Medicina por la Universidad Nacional Mayor de San Marcos en la Facultad de Medicina Humana San Fernando. Generó destacados aportes científicos en tópicos de la Ginecología Oncológica en su estancia por el Hospital Nacional Dos de Mayo y en su Doctorado en Medicina. Le dedicamos el presente artículo científico como un homenaje póstumo a su brillante carrera científico-profesional, gran padre de familia y dedicado humanista con sus pacientes y colegas que ha dejado huella como parte de la historia de la Oncología Ginecológica en el Perú en particular en el Benemérito Hospital Nacional Dos de Mayo.

Además, un especial agradecimiento al Dr José Luis Cabanillas Lapa destacado Médico Cirujano egresado de la Universidad Nacional Mayor de San Marcos con Post Grado en Anatomía Patológica y Post Grado en Oncología Clínica. Ha sido un destacado médico patólogo forense en el Instituto de Medicina Legal Morgue Central de Lima (2000-2004). Asimismo, nuestro agradecimiento al médico patólogo Manuel López-Fuentes, médico asistente del servicio de Anatomía Patológica del Hospital Nacional Dos de Mayo quien en su debido momento contribuyó en la lectura de las láminas histopatológicas, así como en la descripción de la mismas.

De igual modo queremos agradecer a la Bachiller en Derecho y Lingüística de la Universidad Nacional Mayor de San Marcos Bach. Rosa Karina Gonzales Medina maestranda en Derecho del Trabajo y Seguridad Social de la Unidad de Postgrado en la Universidad Nacional Mayor de San Marcos, por sus aportes y corrección de estilo en la redacción científica del presente estudio.

\section{REFERENCIAS BIBLIOGRÁFICAS}

1. Axe SR, Klein VR, Woodruff JD. Choriocarcinoma of the ovary. Obstet Gynecol. julio de 1985;66(1):111-4.

2. Jiao L-Z, Xiang Y, Feng F-Z, Wan X-R, Zhao J, Cui Q-C, et al. Clinical analysis of 21 cases of nongestational ovarian choriocarcinoma. Int J Gynecol Cancer Off J Int Gynecol Cancer Soc. febrero de 2010;20(2):299-302.

3. Lv L, Yang K, Wu H, Lou J, Peng Z. Pure choriocarcinoma of the ovary: a case report. J Gynecol Oncol. 30 de junio de 2011;22(2):135-9.

4. Pectasides D, Pectasides E, Kassanos D. Germ cell tumors of the ovary. Cancer Treat Rev. agosto de 2008;34(5):427-41.

5. Liu X, Zhang X, Pang Y, Ma Y, Zhang X, Liu P. Clinicopathological factors and prognosis analysis of 39 cases of non-gestational ovarian choriocarcinoma. Arch Gynecol Obstet. abril de 2020;301(4):901-12.

6. Koo H-L, Choi J, Kim K-R, Kim J-H. Pure non-gestational choriocarcinoma of the ovary diagnosed by DNA polymorphism analysis. Pathol Int. octubre de 2006;56(10):613-6.

7. Ahn SH, Roh HJ, Cho HJ, You SG, Lee SH, Kwon YS. Pure non-gestational choriocarcinoma arising in the ovary. Eur $\mathrm{J}$ Gynaecol Oncol. agosto de 2016;37(4):549-53.

8. Yamamoto E, Ino K, Yamamoto T, Sumigama S, Nawa A, Nomura $\mathrm{S}$, et al. A pure nongestational choriocarcinoma of the ovary diagnosed with short tandem repeat analysis: case report and review of the literature. Int J Gyne- 
col Cancer Off J Int Gynecol Cancer Soc. febrero de 2007;17(1):254-8.

9. Choi YJ, Chun KY, Kim YW, Ro DY. Pure nongestational choriocarcinoma of the ovary: a case report. World J Surg Oncol. 15 de enero de 2013;11:7.

10. Corakçi A, Ozeren S, Ozkan S, Gürbüz Y, Ustün H, Yücesoy I. Pure nongestational choriocarcinoma of ovary. Arch Gynecol Obstet. febrero de 2005;271(2):176-7.

11. Euscher ED. Germ Cell Tumors of the Female Genital Tract. Surg Pathol Clin. junio de 2019;12(2):621-49.

12. Cheung AN, Zhang HJ, Xue WC, Siu MK. Pathogenesis of choriocarcinoma: clinical, genetic and stem cell perspectives [Internet]. http://dx.doi.org/10.2217/14796694.5.2.217. Future Medicine London, UK; 2009 [citado 19 de mayo de 2021]. Disponible en: https://www.futuremedicine.com/doi/ abs/10.2217/14796694.5.2.217

13. Castiglioni V, Farhang Ghahremani M, Goossens S, De Maglie M, Ardizzone M, Haigh JJ, et al. Immunohistological Description of Nongestational Ovarian Choriocarcinoma in Two Female Mice With Conditional Loss of Trp53 Driven by the Tie2 Promoter. Vet Pathol. julio de 2015;52(4):752-6.

14. Barrière $P$, Mirallie $S$, Jean $M$. Embriología del aparato genital femenino. EMC - Ginecol-Obstet. 1 de enero de 2000;36(4):1-11.

15. Serra Renom I, Vinaixa F, Serra Renom JM, Torres B. Estudio embriológico del aparato genital: aspectos clínicos. Embriological study of the genital apparatus: clinical aspects [Internet]. 1 de mayo de 1987 [citado 20 de mayo de 2021]; Disponible en: http://diposit.ub.edu/dspace/handle/2445/153723

16. Sadler TW, Langman J. Embriologia medica/ Medical Embryology: Con orientacion clinica/ With Clinical Orientation. Ed. Médica Panamericana; 2007. 408 p.

17. Honorio S, Agathanggelou A, Wernert N, Rothe M, Maher ER, Latif F. Frequent epigenetic inactivation of the RASSF1A tumour suppressor gene in testicular tumours and distinct methylation profiles of seminoma and nonseminoma testicular germ cell tumours. Oncogene. enero de 2003;22(3):461-6.

18. Lind GE, Skotheim RI, Lothe RA. The epigenome of testicular germ cell tumors. APMIS Acta Pathol Microbiol Immunol Scand. octubre de 2007;115(10):1147-60.

19. Korkola JE, Houldsworth J, Chadalavada RSV, Olshen AB, Dobrzynski D, Reuter VE, et al. Down-regulation of stem cell genes, including those in a 200-kb gene cluster at 12p13.31, is associated with in vivo differentiation of human male germ cell tumors. Cancer Res. 15 de enero de 2006;66(2):820-7.

20. Skotheim RI, Lind GE, Monni O, Nesland JM, Abeler VM, Fosså SD, et al. Differentiation of human embryonal carcinomas in vitro and in vivo reveals expression profiles relevant to normal development. Cancer Res. 1 de julio de 2005;65(13):5588-98.

21. Korkola JE, Houldsworth J, Dobrzynski D, Olshen AB, Reuter VE, Bosl GJ, et al.

Gene expression-based classification of nonseminomatous male germ cell tumors. Oncogene. 28 de julio de 2005;24(32):5101-7.

22. Santagata S, Ligon KL, Hornick JL. Embryonic stem cell transcription factor signatures in the diagnosis of primary and metastatic germ cell tumors. Am J Surg Pathol. junio de 2007;31(6):836-45.

23. Cheung AN, Shen DH, Khoo US, Wong LC, Ngan HY. p21WAF1/CIP1 expression in gestational trophoblastic disease: correlation with clinicopathological parameters, and
Ki67 and p53 gene expression. J Clin Pathol. febrero de 1998;51(2):159-62.

24. Qiao S, Nagasaka T, Harada T, Nakashima N. p53, Bax and $\mathrm{Bcl}-2$ expression, and apoptosis in gestational trophoblast of complete hydatidiform mole. Placenta. agosto de 1998;19(56):361-9.

25. Fulop V, Mok SC, Genest DR, Gati I, Doszpod J, Berkowitz RS. p53, p21, Rb and mdm2 oncoproteins. Expression in normal placenta, partial and complete mole, and choriocarcinoma. J Reprod Med. febrero de 1998;43(2):119-27.

26. Fulop V, Colitti CV, Genest D, Berkowitz RS, Yiu GK, Ng SW, et al. DOC-2/hDab2, a candidate tumor suppressor gene involved in the development of gestational trophoblastic diseases. Oncogene. 30 de julio de 1998;17(4):419-24.

27. Yazaki-Sun S, Daher S, de Souza Ishigai MM, Alves MTS, Mantovani TM, Mattar R. Correlation of c-erbB-2 oncogene and p53 tumor suppressor gene with malignant transformation of hydatidiform mole. J Obstet Gynaecol Res. junio de 2006;32(3):265-72.

28. Amezcua CA, Bahador A, Naidu YM, Felix JC. Expression of human telomerase reverse transcriptase, the catalytic subunit of telomerase, is associated with the development of persistent disease in complete hydatidiform moles. Am J Obstet Gynecol. junio de 2001;184(7):1441-6.

29. Li HWR, Leung SW, Cheung ANY, Yu MMY, Chan LKY, Wong YF. Expression of maspin in gestational trophoblastic disease. Gynecol Oncol. abril de 2006;101(1):76-81.

30. Inaba $H$, Kawasaki $H$, Hamazaki M, Okugawa T, Uchida K, Honzumi $M$, et al. A case of metastatic ovarian non-gestational choriocarcinoma: successful treatment with conservative type surgery and myeloablative chemotherapy. Pediatr Int Off J Jpn Pediatr Soc. agosto de 2000;42(4):383-5.

31. Chiu RWK, Chim SSC, Wong IHN, Wong CSC, Lee W-S, To $\mathrm{KF}$, et al. Hypermethylation of RASSF1A in human and rhesus placentas. Am J Pathol. marzo de 2007;170(3):941-50.

32. Cheung AN, Zhang DK, Liu Y, Ngan HY, Shen DH, Tsao SW. Telomerase activity in gestational trophoblastic disease. J Clin Pathol. agosto de 1999;52(8):588-92.

33. Wong SY, Ngan HY, Chan CC, Cheung AN. Apoptosis in gestational trophoblastic disease is correlated with clinical outcome and $\mathrm{Bcl}-2$ expression but not Bax expression. Mod Pathol Off J U S Can Acad Pathol Inc. noviembre de 1999;12(11):1025-33.

34. Cameron B, Gown AM, Tamimi HK. Expression of c-erb B-2 oncogene product in persistent gestational trophoblastic disease. Am J Obstet Gynecol. junio de 1994;170(6):1616-21; discussion 1621-1622.

35. Xue WC, Feng HC, Chan KYK, Chiu PM, Ngan HYS, Khoo US, et al. Id helix-loop-helix proteins are differentially expressed in gestational trophoblastic disease. Histopathology. septiembre de 2005;47(3):303-9.

36. Fong P-Y, Xue W-C, Ngan HYS, Chiu P-M, Chan KYK, Tsao $\mathrm{SW}$, et al. Caspase activity is downregulated in choriocarcinoma: a cDNA array differential expression study. J Clin Pathol. febrero de 2006;59(2):179-83.

37. Li HW, Cheung ANY, Tsao SW, Cheung ALM, O WS. Expression of e-cadherin and beta-catenin in trophoblastic tissue in normal and pathological pregnancies. Int J Gynecol Pathol Off J Int Soc Gynecol Pathol. enero de 2003;22(1):63-70.

38. Singh M, Kindelberger D, Nagymanyoki Z, Ng S-W, Quick $\mathrm{CM}$, Elias KM, et al. Matrix metalloproteinases and their in- 
hibitors and inducer in gestational trophoblastic diseases and normal placenta. Gynecol Oncol. julio de 2011;122(1):178-82.

39. Feng $\mathrm{H}$, Cheung ANY, Xue W-C, Wang Y, Wang X, Fu S, et al. Down-regulation and promoter methylation of tissue inhibitor of metalloproteinase 3 in choriocarcinoma. Gynecol Oncol. agosto de 2004;94(2):375-82.

40. Shih IM, Kurman RJ. Expression of melanoma cell adhesion molecule in intermediate trophoblast. Lab Investig J Tech Methods Pathol. septiembre de 1996;75(3):377-88.

41. Arima T, Matsuda T, Takagi N, Wake N. Association of IGF2 and $\mathrm{H} 19$ imprinting with choriocarcinoma development. Cancer Genet Cytogenet. enero de 1997;93(1):39-47.

42. Chilosi M, Piazzola E, Lestani M, Benedetti A, Guasparri I, Granchelli G, et al. Differential expression of p57kip2, a maternally imprinted cdk inhibitor, in normal human placenta and gestational trophoblastic disease. Lab Investig J Tech Methods Pathol. marzo de 1998;78(3):269-76.

43. Siu MKY, Wong ESY, Chan HY, Ngan HYS, Chan KYK, Cheung ANY. Overexpression of NANOG in gestational trophoblastic diseases: effect on apoptosis, cell invasion, and clinical outcome. Am J Pathol. octubre de 2008;173(4):1165-72.

44. Zhang H-J, Siu MK-Y, Wong ES-Y, Wong K-Y, Li AS-M, Chan $\mathrm{KY}-\mathrm{K}$, et al. Oct4 is epigenetically regulated by methylation in normal placenta and gestational trophoblastic disease. Placenta. junio de 2008;29(6):549-54.

45. Li ASM, Siu MKY, Zhang H, Wong ESY, Chan KYK, Ngan HYS, et al. Hypermethylation of SOX2 gene in hydatidiform mole and choriocarcinoma. Reprod Sci Thousand Oaks Calif. septiembre de 2008;15(7):735-44.

46. Chan HY, Siu MKY, Zhang HJ, Wong ESY, Ngan HYS, Chan KYK, et al. Activated Stat3 expression in gestational trophoblastic disease: correlation with clinicopathological parameters and apoptotic indices. Histopathology. agosto de 2008;53(2):139-46.

47. Kurman RJ, editor. Blaustein's Pathology of the Female Genital Tract [Internet]. 4. ${ }^{\text {a }}$ ed. New York: Springer-Verlag; 1994 [citado 20 de mayo de 2021]. Disponible en: https://www. springer.com/gp/book/9781475738896

48. Lazare C, Zhi W, Dai J, Cao C, Sookha RR, Wang L, et al. A pilot study comparing the genetic molecular biology of gestational and non-gestational choriocarcinoma. Am J Transl Res. 2019;11(11):7049-62.
49. Fisher RA, Savage PM, MacDermott C, Hook J, Sebire NJ, Lindsay I, et al. The impact of molecular genetic diagnosis on the management of women with hCG-producing malignancies. Gynecol Oncol. diciembre de 2007;107(3):413-9.

50. Chen F, Tatsumi A, Numoto S. Combined choriocarcinoma and adenocarcinoma of the lung occurring in a man: case report and review of the literature. Cancer. 1 de enero de 2001;91(1):123-9.

51. Anjum AS, Maqsood H, Younus S, Anjum S, Fatima M. A Rare Case of Non-Gestational Metastatic Ovarian Choriocarcinoma: Case Report and Literature Review With a Special Emphasis on Imaging. Cureus. 4 de febrero de 2021;13(2):e13121.

52. Nasioudis D, Chapman-Davis E, Frey MK, Caputo TA, Witkin SS, Holcomb K. Prognostic significance of residual disease in advanced stage malignant ovarian germ cell tumors. Int J Gynecol Cancer Off J Int Gynecol Cancer Soc. marzo de 2019;29(3):554-9.

53. Maestá I, Michelin OC, Traiman P, Hokama P, Rudge MVC. Primary non-gestational choriocarcinoma of the uterine cervix: a case report. Gynecol Oncol. julio de 2005;98(1):146-50.

54. Dehner LP. Gestational and nongestational trophoblastic neoplasia: a historic and pathobiologic survey. Am J Surg Pathol. febrero de 1980;4(1):43-58.

55. Speroff L, Fritz MA. Clinical Gynecologic Endocrinology and Infertility. Lippincott Williams \& Wilkins; 2005. 1398 p.

56. Choi MC, Chung YS, Lee J-W, Kwon BS, Park BK, Kim SI, et al. Feasibility and efficacy of gonadotropin-releasing hormone agonists for the prevention of chemotherapy-induced ovarian insufficiency in patients with malignant ovarian germ cell tumours (KGOG 3048R). Eur J Cancer Oxf Engl 1990. julio de 2020;133:56-65.

Correspondencia:

Carlos Gonzales Medina

Dirección: Alameda Eugenio Yacolevf Edificio 120, Dpto.: 204

Las Torres de San Borja E-mail: dr.carlosgonzales20@gmail.com Teléfono: 996856039 